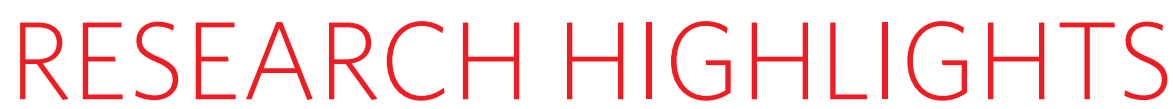

\title{
GENOMICS
}

\section{DNA packaging unravelled}

PLoS Genet. 6, e1000889 (2010)

The largest compartment in the cell's nucleus is the nucleolus, which produces the machinery needed for protein synthesis and regulates other cellular processes. Certain sections of DNA are associated with the nucleolus, but little is known about its molecular structure.

Gernot Längst of the University of Regensburg in Germany and his co-workers analysed DNA from isolated human nucleoli and identified specific genomic regions that are associated with this nuclear compartment.

Analysis of microarray and sequencing data revealed 97 regions, constituting about $4 \%$ of the genome, associated with nucleoli. These regions were enriched for gene families involved in specific functions, such as certain types of immunity. The team hopes that this map will lend insight into how the cell uses DNA-encoded information to construct and maintain structures such as the nucleolus.

\section{ADDICTION}

\section{Junk-food junkies}

Nature Neurosci. doi:10.1038/nn.2519 (2010) Drug addicts' brain reward circuits often exhibit dulled responses, leading the addicts to seek more of the addictive substance to get their fix. Work in rats indicates that fatty foods may trigger similar responses.

Paul Johnson and Paul Kenny at the Scripps Research Institute-Scripps Florida in Jupiter fed rats a high-fat diet, including bacon and sausages, and measured their responsiveness to reward. Animals given prolonged access to the fatty foods needed more stimulation than normal rats to reach a certain reward threshold over time, and gained more weight. Even when the rats were conditioned to associate a light signal with an electric shock to the foot, those with extended access to the high-fat diet continued to eat

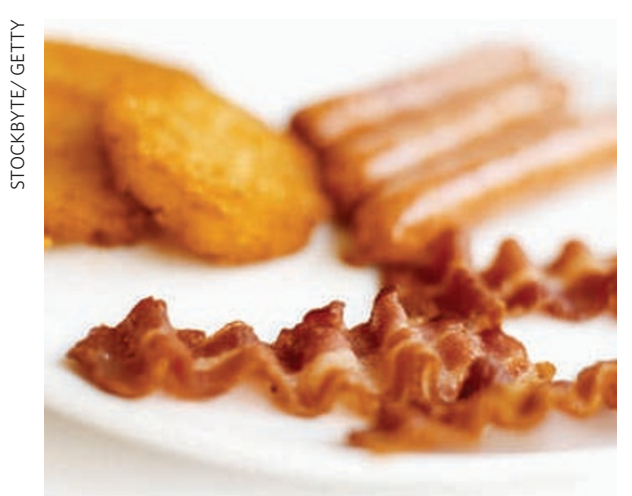

despite seeing the light, indicating the onset of compulsive-eating behaviour.

When the researchers blocked the expression of a dopamine receptor that is downregulated in human drug addicts, rats consuming the rich diet became compulsive eaters more rapidly.

\section{PHOTONICS}

\section{Carbon light catcher}

Nature Photon. doi:10.1038/nphoton.2010.40 (2010) Physicists and engineers are excited about graphene - a single layer of carbon atoms because of its electrical and light-conducting properties. Thomas Mueller, Fengnian Xia and Phaedon Avouris at the IBM Thomas J. Watson Research Center in Yorktown Heights, New York, have used graphene to build a device that detects light and converts it into an electrical signal.

Their fast photodetector could pick up light pulses with a wavelength of 1.55 micrometres and introduced no errors in data transmission in a 10-gigabit-per-second optical data link. The authors say that because graphene photodetectors can operate at high speeds and are sensitive to a range of wavelengths, they could have applications in fields such as communications and remote sensing.

\section{NEURODEVELOPMENT}

\section{Baby talk}

Neuron 65, 852-858 (2010)

Infants just seven months old have a specialized sensitivity to the human voice and its emotional content that is similar to that of adults.

Tobias Grossmann at Birkbeck, University of London, and his colleagues used nearinfrared spectroscopy to monitor the activity of different brain areas in four- and sevenmonth-old babies as they listened to various recorded voices and sounds.

Compared with other sounds, the researchers found increased responses to the sound of a human voice in the right and left superior temporal cortex by the age of seven months. They saw a greater response in a 